\title{
Cylindrical Langmuir probes beyond the orbital-motion-limited regime
}

R. D. Estes

Harvard-Smithsonian Center for Astrophysics, 60 Garden St., Cambridge, Massachusetts 02138

\section{J. R. Sanmartín ${ }^{\text {a) }}$}

Escuela Técnica Superior de Ingenieros Aeronáuticos, Universidad Politécnica de Madrid, 28040 Madrid, Spain

(Received 5 April 2000; accepted 12 June 2000)

\begin{abstract}
The current $I$ to a cylindrical probe at rest in an unmagnetized plasma, with probe bias highly positive, is determined. The way $I$ lags behind the orbital-motion-limited (OML) current, $I_{\mathrm{OML}}$ $\propto R$, as the radius $R$ exceeds the maximum radius for the OML regime to hold, is of interest for space-tether applications. The ratio $I / I_{\mathrm{OML}}$ is roughly a decreasing function of $R / \lambda_{\mathrm{De}}-R_{\max } / \lambda_{\mathrm{De}}$, which is independent of bias, with $\lambda_{\mathrm{De}}$ the electron Debye length and $R_{\max } / \lambda_{\mathrm{De}}$ roughly an increasing function of the temperature ratio, $T_{i} / T_{e}$. The dependence of current on ion energy is used to discuss the effect of probe motion through the plasma, a case applying to tethers in low orbit.

(C) 2000 American Institute of Physics. [S1070-664X(00)00210-X]
\end{abstract}

\section{INTRODUCTION}

The interaction of conductive space tethers with the magnetized ionosphere has potential applications that range from power generation and propulsion (or drag for deorbiting purposes), ${ }^{1,2}$ to the use of wave and particle emissions. ${ }^{3-5}$ The basic problem is how to collect ionospheric electrons: The small electron gyroradius and Debye length could greatly reduce collection through magnetic guiding and electric shielding. Using the thin tether itself (left bare over kilometers of its length) as the anode offers the benefits of (1) passive electron collection over a large area with no shielding or magnetic effects ${ }^{6}$ and (2) relative insensitivity to regular drops in plasma density along its orbit. ${ }^{7}$ A National Aeronautics and Space Administration experiment (Propulsive Small Expendable Deployer System) will test bare-tether collection this year in a Delta-2 orbital flight. Bare tethers are being considered for reboost of both the Russian MIR and the International Space Stations, as well as for future use in the Jovian system.

A bare tether collects current as a cylindrical Langmuir probe. The electron current $I$ to a long cylinder at rest in a collisionless, unmagnetized, Maxwellian plasma of density $N_{\infty}$ and temperatures $T_{e}$ and $T_{i}$, may be written as

$$
I=I_{\mathrm{th}} \times \mathrm{a} \text { function of } e \Phi_{P} / k T_{e}, R / \lambda_{\mathrm{De}}, T_{i} / T_{e},
$$

where $I_{\mathrm{th}} \equiv 2 \pi R L \times e N_{\infty} \times \sqrt{k T_{e} / 2 \pi m_{e}}$ is the random current, $\lambda_{\mathrm{De}}$ is the Debye length, and $R, L$, and $\Phi_{P}$ are probe radius, length, and bias. For cylinders thin enough, however, $I / I_{\text {th }}$ only depends on $e \Phi_{P} / k T_{e}$. This is the orbital-motionlimited (OML) regime; at high bias $\left(e \Phi_{P} / k T_{e} \sim 10^{3}\right.$ for a typical tether) one has

$$
\begin{aligned}
I_{\mathrm{OML}} & \approx I_{\mathrm{th}} \times \sqrt{4 e \Phi_{P} / \pi k T_{e}} \\
& =2 R L e N_{\infty} \sqrt{2 e \Phi_{P} / m_{e}} \quad\left(e \Phi_{P} \gg k T_{e}\right) .
\end{aligned}
$$

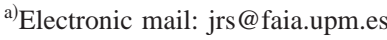

Actually, this equation is also valid for a noncircular cross section if convex enough, with $1 / \pi$ times its perimeter replacing $2 R$ above. ${ }^{8}$

Since $I_{\mathrm{OML}}$ is proportional to radius (or perimeter) of the cross section, a large current may require a large radius; if this is too large, however, the current $I$ will not reach the OML value because of electrical effects. The maximum radius $R_{\max }$ for the OML regime to hold with other parameters fixed was determined recently. ${ }^{9}$ The maximum width of a thin tape and conditions to have negligible effects from the magnetic field, and from electrons trapped in bounded orbits, were also established in Ref. 9. Here we study the way $I / I_{\mathrm{OML}}$ drops below unity when $R$ goes above $R_{\max }$ at very high bias, a matter of interest for the design of bare tethers. In Sec. II we recollect the basic structure of the analysis in Ref. 9, and point out the new features of the present problem. In Sec. III we describe the scheme to calculate the current. In Sec. IV we discuss results on $I / I_{\mathrm{OML}}$; its dependence on $R_{\text {max }}$, which varies with plasma parameters; and the effect of tether motion relative to the ionosphere.

\section{STRUCTURE OF THE POTENTIAL}

In general, determining electron trajectories to obtain the collected current $I$ requires solving Poisson's equation for $\Phi(r)$

$$
\frac{\lambda_{D i}^{2}}{r} \frac{d}{d r} r \frac{d}{d r}\left(\frac{e \Phi}{k T_{i}}\right)=\frac{N_{e}}{N_{\infty}}-\frac{N_{i}}{N_{\infty}} \approx \frac{N_{e}}{N_{\infty}}-\exp \left(-\frac{e \Phi}{k T_{i}}\right),
$$

with boundary conditions $\Phi=\Phi_{P}>0$ at $r=R, \Phi \rightarrow 0$ as $r$ $\rightarrow \infty$. The Boltzmann law used for the ion density $N_{i}$ is quite accurate at the high bias of interest. Regarding $N_{e}$, since the Vlasov equation conserves the distribution function $f_{e}(\mathbf{r}, \mathbf{v})$ along orbits, and trapped electrons may be ignored, ${ }^{9}$ we have 


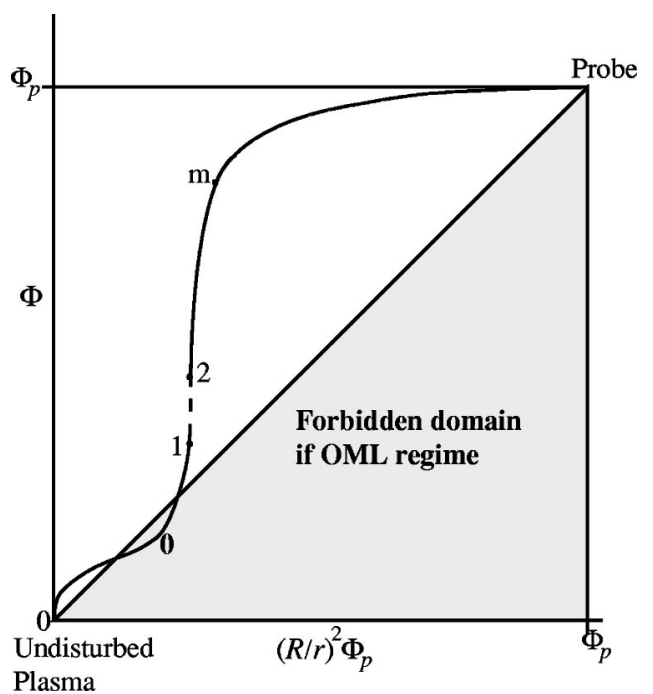

FIG. 1. Schematics of potential $\Phi$ vs $\Phi_{P} R^{2} / r^{2}$ for $R>R_{\max }$ (maximum radius for the OML regime to hold). The plasma is quasineutral below point 1 , with no potential barriers below point 0 . The ratio $\Phi r^{2} / \Phi_{P} R^{2}$ reaches a large maximum at a point $m$ in the ion-free, broad region above thin, nonquasineutral layers at points 1 and 2.

$f_{e}(\mathbf{r}, \mathbf{v})=f_{e M}\left(v_{\infty}\right)$ (undisturbed Maxwellian) if the $\mathbf{r}, \mathbf{v}$ orbit traced back in time reaches infinity and $f_{e}(\mathbf{r}, \mathbf{v})=0$ otherwise. ${ }^{10}$ Since energy is also conserved and $f_{e M}$ is isotropic, values for $\mathbf{r}, \mathbf{v}$ determine the value of $f_{e M}$ in terms of the local potential $\Phi(r)$.

Both density $N_{e}$ at any radius $r$ and current $I$ can then be written as integrals of $f_{e M}$ over axial velocity and (allowed ranges of) angular momentum $J$ and energy $E$ in the perpendicular plane, which are all three conserved. Defining

$$
\begin{aligned}
& J_{r}(E) \equiv \sqrt{2 m_{e} r^{2}[E+e \Phi(r)]}, \\
& J_{r}^{*}(E) \equiv \operatorname{minimum}\left[J_{r^{\prime}}(E) ; r^{\prime} \geqslant r\right],
\end{aligned}
$$

one finally arrives at ${ }^{9}$

$$
\begin{aligned}
\frac{N_{e}}{N_{\infty}}= & \int_{0}^{\infty} \frac{d E}{\pi k T_{e}} \exp \left(\frac{-E}{k T_{e}}\right) \\
& \times\left[2 \sin ^{-1} \frac{J_{r}^{*}(E)}{J_{r}(E)}-\sin ^{-1} \frac{J_{R}^{*}(E)}{J_{r}(E)}\right], \\
I= & \frac{2 L e N_{\infty}}{m_{e}} \times \int_{0}^{\infty} \frac{d E}{k T_{e}} \exp \left(\frac{-E}{k T_{e}}\right) J_{R}^{*}(E) .
\end{aligned}
$$

For given $r$ and $E$, values $J=0$ and $J=J_{r}(E)$ correspond to zero azimuthal and radial velocities, respectively. In case we have $J_{r}^{*}(E)<J_{r}(E)$, inward trajectories in the range $J_{r}^{*}(E)$ $<J<J_{r}(E)$ are unpopulated; such electrons, if traced back in time, turn around at radii between $r$ and the (larger) radius where the minimum in (4) occurs: There is a barrier in the radial effective potential energy. Note that $N_{e}$ is a functional of the full potential structure rather than a function of its local value. That structure, as determined in Ref. 9, can be illustrated by schematically displaying $\Phi$ versus $\Phi_{P} R^{2} / r^{2}$, with the ordinate-to-abscissa ratio proportional to $r^{2} \Phi$ (Fig. 1).

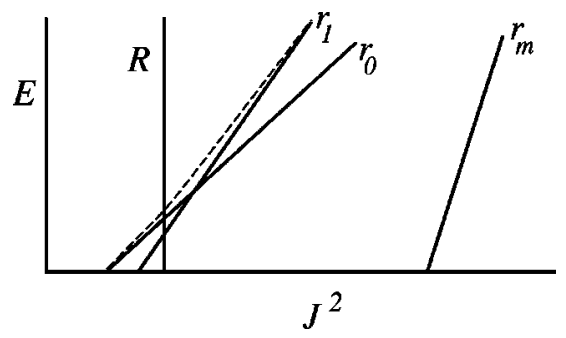

FIG. 2. Straight lines in the $E$ (energy) vs $J^{2}$ (squared angular momentum) plane, for the $r$-family defined in Eq. (10), $J^{2}=J_{r}^{2}(E)$. Shown are $r$-lines for the probe and for points $m, 1$, and 0 in Fig. 1, as well as the envelope of lines in the $r_{0}-r_{1}$ range.

The faraway quasineutral solution to (2), $N_{e} \approx N_{i}$, behaves as $\Phi \sim 1 / r$. As one moves up on the profile from the origin in the figure, $r^{2} \Phi(r)$ decreases to a minimum at some radius $r_{0}$. The quasineutral solution remains valid further above, up to a point 1 where $-d \Phi / d r$ diverges. Above point 1 there are two thin nonquasineutral layers that take the solution to values $\Phi_{1} \ll \Phi \ll \Phi_{P}$, and to a radius $r_{2}$ a bit closer to the probe; points $0-1$ if drawn to scale would lie very close to the origin in Fig. 1 because $e \Phi_{0}$ and $e \Phi_{1}$ are of order of $k T_{i}$ whereas $e \Phi_{P} / k T_{i}$ is very large. In the broad region between radii $r_{2}$ and $R$ the ion density is negligible, and $r^{2} \Phi(r)$ reaches a large maximum at some point $m$ before dropping to $R^{2} \Phi_{P}$ at the probe.

Note in Eqs. (3) and (4) that, for any particular $r$, having $J_{r}^{*}(0)=J_{r}(0)$ suffices to make $J_{r}^{*}(E)$ equal to $J_{r}(E)$ for all $E \geqslant 0$; using $J_{r}^{2}(0) \propto r^{2} \Phi(r)$, the no-barrier condition reads

$$
r^{\prime 2} \Phi\left(r^{\prime}\right) \geqslant r^{2} \Phi(r), \quad r^{\prime} \geqslant r .
$$

Since we have

$$
d\left(r^{2} \Phi\right) / d r>0, \quad r>r_{0},
$$

there are energy barriers at no radius below point 0 in Fig. 1

$$
J_{r}^{*}(E)=J_{r}(E) \text { for } E \geqslant 0 \quad\left(r \geqslant r_{0}\right) .
$$

Again, this can be illustrated by depicting the structure of the $r$-family of straight lines

$$
J^{2}=J_{r}^{2}(E) \quad \text { or } E=\frac{J^{2}}{2 m_{e} r^{2}}-e \Phi(r),
$$

in the $J^{2}-E$ plane (Fig. 2). The line slope steepens monotonically as $r$ decreases, moving up in Fig. 1, while the foot, $J^{2}(E=0)$, varies as $r^{2} \Phi(r)$. Property (8) means that, throughout the range $r>r_{0}$, the $r$-line moves to the left in Fig. 2 for all positive energies. Past point 0 , however, the line foot moves back to the right. Since we have $r_{1}^{2} \Phi_{1}$ $>r_{0}^{2} \Phi_{0}$ and $r_{1}<r_{0}$, the $r_{0}$ - and $r_{1}$-lines meet at some positive energy, resulting in an $r$-dependent energy range with effective potential energy barriers.

The envelope $J^{2}=J_{\text {env }}^{2}(E)$ of lines in the range $r_{1}<r$ $<r_{0}$ is determined by the equations $J^{2}-J_{r}^{2}(E)=0, \partial\left[J^{2}\right.$ $\left.-J_{r}^{2}(E)\right] / \partial r=0$, yielding the parametric representation

$$
\begin{aligned}
& J^{2}=J_{\text {env }}^{2}(r) \equiv-m_{e} r^{3} e d \Phi / d r, \\
& E=E_{\text {env }}(r) \equiv-e \Phi(r)-r e d \Phi / d r .
\end{aligned}
$$


The envelope lies to the left of all lines in that range, touching each line at the point given by Eqs. (11a) and (11b); it leaves the $r_{0}$-line at $E=0,{ }^{11}$ and reaches the $r_{1}$-line asymptotically, as $E_{\text {env }}$ and $J_{\text {env }}^{2}$ diverge with $-d \Phi / d r$ as $r \rightarrow r_{1}$ (Fig. 2). For each radius between $r_{0}$ and $r_{1}$ only that part of the envelope below the touching point enters in the determination of $J_{r}^{*}(E)$; we would thus have $J_{r}^{*}(E)=J_{\text {env }}(E)$ for $E<E_{\mathrm{env}}(r)$, and $J_{r}^{*}(E)=J_{r}(E)$ otherwise. As $r$ approaches $r_{1}$, however, $E_{\text {env }}(r)$ diverges making $J_{r}^{*}(E)=J_{\text {env }}(E)$ valid for all energies. As $\Phi$ rises rapidly with decreasing $r$ above point 1 in Fig. 1, the line foot in Fig. 2 moves far to the right, the line itself steepening moderately. Within thin layers and broad region we would then have

$$
J_{r}^{*}(E)=J_{\text {env }}(E) \text { for } E \geqslant 0 \quad\left(r \leqslant r_{1}\right) .
$$

At point $m$ in Fig. 1, the line foot turns again to the left, finally ending at the $R$-line (Fig. 2), which is near-vertical $\left(R \ll r_{1}, E \sim k T_{e} \ll e \Phi_{P}\right)$, and has its foot to the right of the envelope, corresponding to point 0 lying below the diagonal in Fig. 1. Clearly, Eq. (12) fails in some neighborhood of the probe. At $R$ in particular, we have

$$
\begin{aligned}
& J_{R}^{*}(E)=J_{\text {env }}(E) \text { for } 0<E<E_{c}, \\
& J_{R}^{*}(E)=J_{R}(E) \text { for } E>E_{c},
\end{aligned}
$$

with $E_{c}$ the energy at the crossing of envelope and $R$-line; this results in a ratio $I / I_{\mathrm{OML}}<1$, or $R>R_{\max }$. Note that maximum (OML) current in (6) would only occur with $J_{R}^{*}(E)$ $=J_{R}(E)$ for all energies (no effective energy barrier for $R$ ), a condition requiring, according to (7)

$$
r^{2} \Phi(r) \geqslant R^{2} \Phi_{p}, \quad r \geqslant R .
$$

Point 0 would then lie at or above the diagonal in Fig. 1, and the entire $R$ line would appear to the left of the envelope in Fig. $2\left(R \leqslant R_{\max }\right)$; with $E \ll e \Phi_{P}$ we would have $J_{R}(E)$ $\approx J_{R}(0)$, (6) then recovering Eq. (1) for the high-bias OML law.

\section{CALCULATION OF CURRENT}

Using (1) and (6), the ratio $I / I_{\mathrm{OML}}$ takes the form

$$
\frac{I}{I_{\mathrm{OML}}}=\int_{0}^{\infty} \frac{d E}{k T_{e}} \exp \left(\frac{-E}{k T_{e}}\right) \frac{J_{R}^{*}(E)}{J_{R}(0)},
$$

with $J_{R}^{*}(E)$ given by Eqs. (13a) and (13b), and $J_{R}(E)$ $=J_{R}(0)=\sqrt{2 m_{e} R^{2} e \Phi_{P}}$. The integral above, therefore, must be split into separate integrals for intervals $0<E<E_{c}$ and $E>E_{c}$. In the first interval one needs $J_{\text {env }}(E)$, which involves the structure of the potential in a narrow radial range. Since the envelope is tangent to both $r_{0}$ and $r_{1}$ lines, a simple but accurate approximation for $J_{\text {env }}(E)$ can be readily obtained without actually knowing $\Phi(r)$

$$
J_{\text {env }}^{2}(E)=J_{r_{1}}^{2}(E)-\frac{2 m_{e}\left(r_{1}^{2} e \Phi_{1}-r_{0}^{2} e \Phi_{0}\right)^{2}}{r_{1}^{2} e \Phi_{1}-r_{0}^{2} e \Phi_{0}+\left(r_{0}^{2}-r_{1}^{2}\right) E} .
$$

We still need to solve Eq. (2) because the values $r_{0}, \Phi_{0}, r_{1}$, and $\Phi_{1}$ are unknown and depend on the entire potential structure.

In Eq. (5) for the electron density we use (13a) and (13b) for $J_{R}^{*}(E)$, and appropriate expressions for $J_{r}^{*}(E)$ at differ- ent $r$ values or ranges. First, the quasineutrality relation $N_{e}$ $=N_{i}$ at point 0 , with $J_{r}^{*}(E)$ taken from (9), yields

$$
\exp \left(-\frac{e \Phi_{0}}{k T_{i}}\right)=1-\int_{0}^{\infty} \frac{d E}{\pi k T_{e}} \exp \left(\frac{-E}{k T_{e}}\right) \sin ^{-1} \frac{J_{R}^{*}(E)}{J_{r_{0}}(E)} .
$$

Again, the quasineutrality relation at point 1 , with $J_{r}^{*}(E)$ from (12), yields

$$
\begin{aligned}
\exp \left(-\frac{e \Phi_{1}}{k T_{i}}\right)= & \int_{0}^{\infty} \frac{d E}{\pi k T_{e}} \exp \left(\frac{-E}{k T_{e}}\right) \\
& \times\left[2 \sin ^{-1} \frac{J_{\mathrm{env}}(E)}{J_{r_{1}}(E)}-\sin ^{-1} \frac{J_{R}^{*}(E)}{J_{r_{1}}(E)}\right] .
\end{aligned}
$$

Since Eq. (12) holds in some neighborhood of point 1, the derivative of the quasineutrality relation with respect to $\Phi$ at $r_{1}$ (where $d r / d \Phi$ vanishes) finally gives

$$
\begin{aligned}
\exp \left(\frac{-e \Phi_{1}}{k T_{i}}\right)= & \int_{0}^{\infty} \frac{T_{i} \exp \left(-E / k T_{e}\right) d E}{2 \pi T_{e}\left(E+e \Phi_{1}\right)} \\
& \times\left[2 \frac{J_{\text {env }}(E)}{\sqrt{J_{r_{1}}^{2}(E)-J_{\text {env }}^{2}(E)}}\right. \\
& -\frac{J_{R}^{*}(E)}{\left.\sqrt{J_{r_{1}}^{2}(E)-J_{R}^{*}(E)}\right] .} .
\end{aligned}
$$

Note that the integrals in Eqs. (17)-(19) [and later integrals also involving $\left.J_{R}^{*}(E)\right]$ must each be split into energy ranges below and above $E_{c}$, as with (15). Those equations, together with the relation defining $E_{c}$

$$
J_{\text {env }}\left(E_{c}\right)=J_{R}\left(E_{c}\right) \approx J_{R}(0),
$$

serve to determine $e \Phi_{0} / k T_{i}, \quad e \Phi_{1} / k T_{i}, \quad \sigma_{1}$ $\equiv e \Phi_{P} R^{2} / k T_{i} r_{1}^{2}$, and $r_{1} / r_{0}$ as functions of $T_{e} / T_{i}$ and $E_{c} / k T_{e}$. Equation (15) now gives

$$
I / I_{\mathrm{OML}}=\text { a function of } T_{e} / T_{i}, \quad E_{c} / k T_{e} .
$$

One could then obtain $e \Phi_{0} / k T_{i}, e \Phi_{1} / k T_{i}, \sigma_{1}$, and $r_{1} / r_{0}$ as functions of $T_{e} / T_{i}$ and $I / I_{\mathrm{OML}}$.

Since the quasineutral solution is singular at $r_{1}$, the lefthand-side of Eq. (2) must be retained in a thin layer above point 1 in Fig. 1, with charge densities expanded around point-1 values. ${ }^{9}$ At a radius $r_{2}$ close to $r_{1}$ the potential itself blows up to infinity, requiring a second nonquasineutral thin layer that just allows a smooth match to the solution in the broad region reaching to the probe. An analysis of the first layer as in Ref. 9 yields

$$
\sigma_{2}=\sigma_{1}\left[1+6.9\left(\frac{2 \sigma_{1}^{2}}{\lambda \mu}\right)^{1 / 5}\left(\frac{\lambda_{\mathrm{Di}}}{R}\right)^{4 / 5}\left(\frac{k T_{i}}{e \Phi_{P}}\right)^{2 / 5}\right],
$$

with $\sigma_{2} \equiv \sigma_{1} \times r_{1}^{2} / r_{2}^{2}$, and $\mu$ and $\lambda$ new functions of $T_{e} / T_{i}$ and $E_{c} / K T_{e}$ 


$$
\begin{aligned}
\mu \equiv & \int_{0}^{\infty} \frac{d E}{\pi k T_{e}} \exp \left(\frac{-E}{k T_{e}}\right)\left[\frac{2 J_{\mathrm{env}}(E)}{\sqrt{J_{r_{1}}^{2}(E)-J_{\mathrm{env}}^{2}(E)}}\right. \\
& \left.-\frac{J_{R}^{*}(E)}{\sqrt{J_{r_{1}}^{2}(E)-J_{R}^{*}(E)}}\right], \\
\lambda \equiv & -\exp \left(-\frac{e \Phi_{1}}{k T_{i}}\right)+\int_{0}^{\infty} \frac{k T_{i}^{2} d E \exp \left(-E / k T_{e}\right)}{4 \pi T_{e}\left(E+e \Phi_{1}\right)^{2}} \\
& \times\left[2 J_{\mathrm{env}}(E) \frac{3 J_{r_{1}}^{2}(E)-2 J_{\mathrm{env}}^{2}(E)}{\left(J_{r_{1}}^{2}(E)-J_{\mathrm{env}}^{2}(E)\right)^{3 / 2}}\right. \\
& \left.-J_{R}^{*}(E) \frac{3 J_{r_{1}}^{2}(E)-2 J_{R}^{*^{2}}(E)}{\left(J_{r_{1}}^{2}(E)-J_{R}^{*^{2}}(E)\right)^{3 / 2}}\right] .
\end{aligned}
$$

In the broad region above the thin layers we take $e \Phi$ $\gg e \Phi_{1} \sim k T_{i}, e \Phi \gg E \sim k T_{e}$ (Fig. 1), making $N_{i} / N_{\infty}$ negligibly small in Eq. (2). Also, since $r$-lines now lie far to the right in Fig. 2 throughout most of this region, we simplify the integral for $N_{e} / N_{\infty}$ in (5) by using Eq. (12) and the approximations $\quad J_{R}^{*}(E) \sim J_{\text {env }}(E) \ll J_{r}(E), \quad J_{r}(E) \approx J_{r}(0)$, leading to

$$
\begin{aligned}
& \frac{N_{e}}{N_{\infty}} \approx \frac{\kappa}{\pi} \frac{R}{r} \sqrt{\frac{\Phi_{P}}{\Phi}} \\
& \kappa \equiv \int_{0}^{\infty} \frac{d E}{k T_{e}} \exp \left(\frac{-E}{k T_{e}}\right)\left[2 \frac{J_{\text {env }}(E)}{J_{R}(0)}-\frac{J_{R}^{*}(E)}{J_{R}(0)}\right],
\end{aligned}
$$

with $\kappa$ again a function of $T_{e} / T_{i}$ and $E_{c} / k T_{e}$. Note that use of $J_{r}(E) \approx J_{r}(0)$ and (12) fails near $r_{1}$ and $R$ respectively, overestimating $N_{e}$, whereas taking $J_{R}^{*} / J_{r}$ and $J_{\text {env }} / J_{r}$ small underestimates $N_{e}$ and fails near both $r_{1}$ and $R$. In the $R$ $=R_{\max }\left[J_{R}^{*}(E)=J_{R}(E)\right]$ case of Ref. 9 the exact value for $N_{e}(r=R)$ in Eq. (5) is $(1 / 2) N_{\infty}$, while the approximation in Eq. (24) gives $N_{\infty} \times \kappa / \pi$, which Fig. 5(b) of Ref. 9 shows to be about $N_{\infty}$ (for $T_{e}=T_{i}$ ); a net overestimate of $N_{e}$ increases shielding and leads to an underestimate of both $R_{\max }$ and $I / I_{\mathrm{OML}}\left(R>R_{\max }\right)$. Clearly, the error will be greater the lower the bias. ${ }^{12}$

Using $N_{i}=0$ and Eqs. (24) and (25) in (2), and defining

$$
u \equiv \ln \frac{r_{2}}{r}, \quad g \equiv\left[\pi \frac{\sqrt{\sigma_{2}}}{\kappa} \frac{\lambda_{\mathrm{Di}}^{2}}{R^{2}} \frac{k T_{i}}{e \Phi_{P}}\right]^{2 / 3} \frac{e \Phi}{k T_{i}},
$$

Poisson's equation, and the boundary conditions imposed by matching to the second layer, become

$$
\frac{d^{2} g}{d u^{2}}=\frac{e^{-u}}{\sqrt{g}}, \quad g=\frac{d g}{d u}=0 \quad \text { at } \quad u=0 \quad\left(g \propto u^{4 / 3}\right) .
$$

From the numerical solution for $g(u)$ one finds $\Phi(r)$; the boundary condition $\Phi=\Phi_{P}$ at $r=R$ then yields

$$
g\left[\ln \left(\sqrt{\frac{e \Phi_{P}}{k T_{i} \sigma_{2}}}\right)\right]=\left(\frac{\pi^{2} \sigma_{2}}{\kappa^{2}}\right)^{1 / 3}\left(\frac{\lambda_{\mathrm{Di}}}{R}\right)^{4 / 3}\left(\frac{e \Phi_{P}}{k T_{i}}\right)^{1 / 3} .
$$

Using (21) in (26) determines a last relation

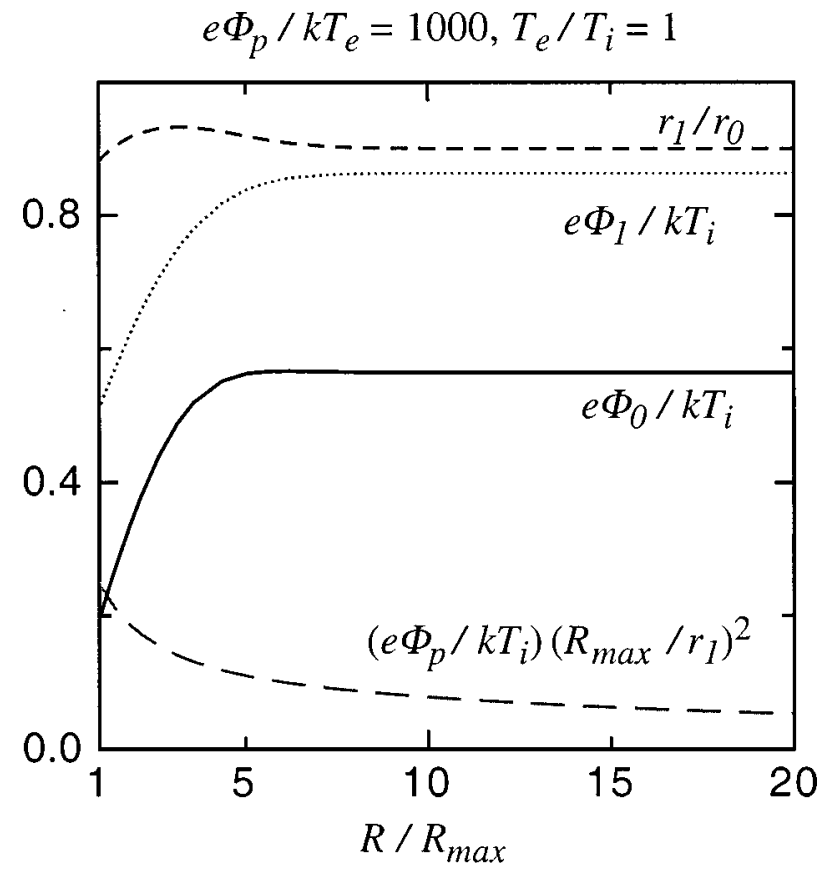

FIG. 3. Dimensionless ratios $e \Phi_{0} / k T_{i}, \quad e \Phi_{1} / k T_{i}, \quad \sigma_{1} R_{\max }^{2} / R^{2}$ $\equiv e \Phi_{P} R_{\max }^{2} / k T_{i} r_{1}^{2}$, and $r_{1} / r_{0}$ vs $R / R_{\max }$, for $T_{e} / T_{i}=1$ and $e \Phi_{P} / k T_{e}$ $=1000$; results for $e \Phi_{P} / k T_{e}=300$ and 3000 are quite similar.

$$
\frac{E_{c}}{k T_{e}}=\text { a function of } \frac{T_{e}}{T_{i}}, \frac{\lambda_{\mathrm{Di}}}{R}, \frac{e \Phi_{P}}{k T_{i}} .
$$

Equations $\left(15^{\prime}\right)$ and (27) give $I / I_{\mathrm{OML}}$ versus $e \Phi_{P} / k T_{e}$, $R / \lambda_{\text {De }}$, and $T_{e} / T_{i}$.

The $R=R_{\max }$ limit studied in Ref. 9 corresponds to $E_{c}$ $=0$, Eq. (15) or $\left(15^{\prime}\right)$ then giving $I=I_{\mathrm{OML}}$. Using (16), Eq. (20) would read $J_{\text {env }}(0)=J_{R}(0)$ or $r_{0}^{2} \Phi_{0}=R^{2} \Phi_{P}$ (point 0 on the diagonal of Fig. 1); this condition, together with Eqs. (17)-(19), determined $\sigma_{1}, e \Phi_{1} / k T_{i}$, and $e \Phi_{0} / k T_{i}$ ( $=\sigma_{1} r_{1}^{2} / r_{0}^{2}$ ) as functions of just $T_{e} / T_{i}$. With $\mu, \lambda$, and $\kappa$ functions of $T_{e} / T_{i}$ too, Eq. (27) gave $R_{\max } / \lambda_{\mathrm{De}}$ versus $e \Phi_{P} / k T_{e}$ and $T_{e} / T_{i}$ (Fig. 7 in Ref. 9).

For completeness, we now consider briefly the OML regime $\left(R / R_{\max } \leqslant 1\right)$, which is naturally of less interest because the current is known, $I=I_{\mathrm{OML}}$. For the non-OML conditions we have studied until now, the potential profile below point 0 in Fig. 1 [which was determined by the equation $N_{i}=N_{e}$ with $J_{r}^{*}(E)=J_{r}(E)$ in (5)] varied with $J_{R}^{*}(E)$, and thus with $R / R_{\max }$. In the OML regime, however, we have $J_{R}^{*}(E)$ $=J_{R}(E)$, and thus a single profile throughout. As $R / R_{\max }$ decreases from unity, point 0 just moves down on that particular profile away from the diagonal, with $R^{2} \Phi_{P} / r_{0}^{2} \Phi_{0}$ decreasing too. For specified $T_{e} / T_{i}$ and $R^{2} \Phi_{P} / r_{0}^{2} \Phi_{0}$ values, Eqs. (17)-(19) would give $\sigma_{1}, e \Phi_{1} / k T_{i}, e \Phi_{0} / k T_{i}$, and $r_{1} / r_{0}$; then (22), (23), and (25) give $\mu, \lambda$, and $\kappa$. Finally, using (21) in (26) would yield $R^{2} \Phi_{P} / r_{0}^{2} \Phi_{0}$ versus $R / \lambda_{\mathrm{De}}$, $T_{e} / T_{i}$, and $e \Phi_{P} / k T_{e}$.

\section{DISCUSSION OF RESULTS}

Figure 3 shows $e \Phi_{0} / k T_{i}, e \Phi_{1} / k T_{i}, \quad r_{1} / r_{0}$ and $e \Phi_{P} R_{\max }^{2} / k T_{i} r_{1}^{2}\left(\equiv \sigma_{1} R_{\max }^{2} / R^{2}\right)$ versus $R / R_{\max }$ at $T_{e} / T_{i}=1$ and $e \Phi_{P} / k T_{e}=1000 ; \sigma_{1} R_{\max }^{2} / R^{2}$ was plotted because $\sigma_{1}$ 

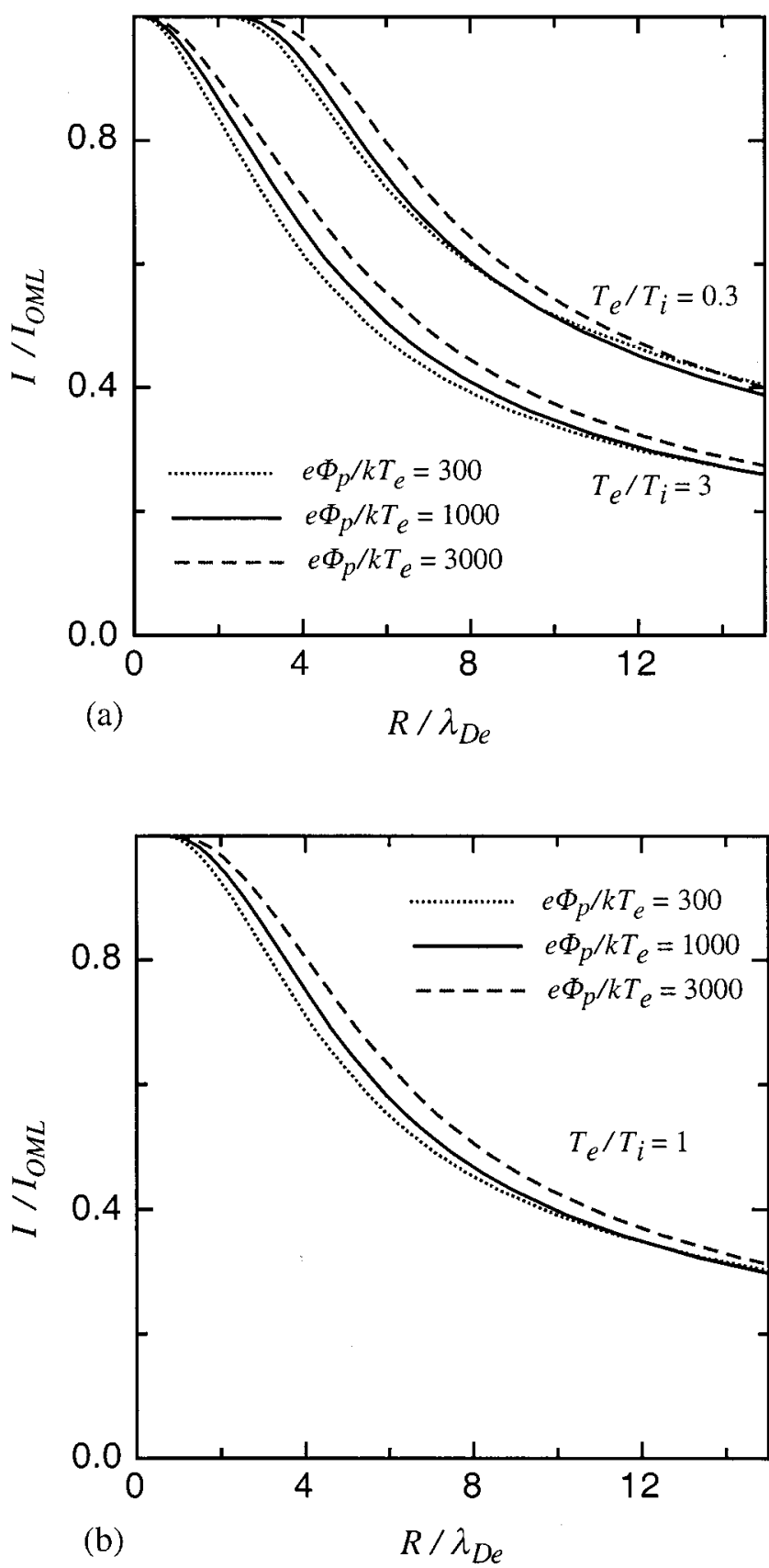

FIG. 4. Current ratio $I / I_{\mathrm{OML}}$ vs $R / \lambda_{\mathrm{De}}$ for a few values of $e \Phi_{P} / k T_{e}$ and (a) $T_{e} / T_{i}=0.3$ and 3 , (b) $T_{e} / T_{i}=1$.

gets too large. As suggested by other results below, values for $e \Phi_{P} / k T_{e}=300,1000$, and 3000 are quite close to each other. Actually, as noted in Sec. III, plots in Fig. 3 would be fully $e \Phi_{P} / k T_{e}$-independent if $I / I_{\mathrm{OML}}$ replaced $R / R_{\max }$.

Figures 4(a) and 4(b) give $I / I_{\mathrm{OML}}$ versus $R / \lambda_{\mathrm{De}}$ for a few values of $T_{e} / T_{i}$ and $e \Phi_{P} / k T_{e}$. Note that the dependence on probe bias is indeed very weak. We also note that previous asymptotic results on the limit $R / \lambda_{\mathrm{De}} \rightarrow \infty$, at $T_{e} / T_{i}=1$, showed $I / I_{\mathrm{OML}}$ to approach a limit value that decreased with increasing $e \Phi_{P} / k T_{e}{ }^{13}\left(I / I_{\mathrm{OML}} \rightarrow 1.29 k T_{e} / e \Phi_{P}\right.$, here being 0.075, 0.041, and 0.024 for $e \Phi_{P} / k T_{e}=300,1000$, and 3000, respectively). Crossover points for the curves can be seen in Figs. 4(a) and 4(b), however. The asymptotic ap-

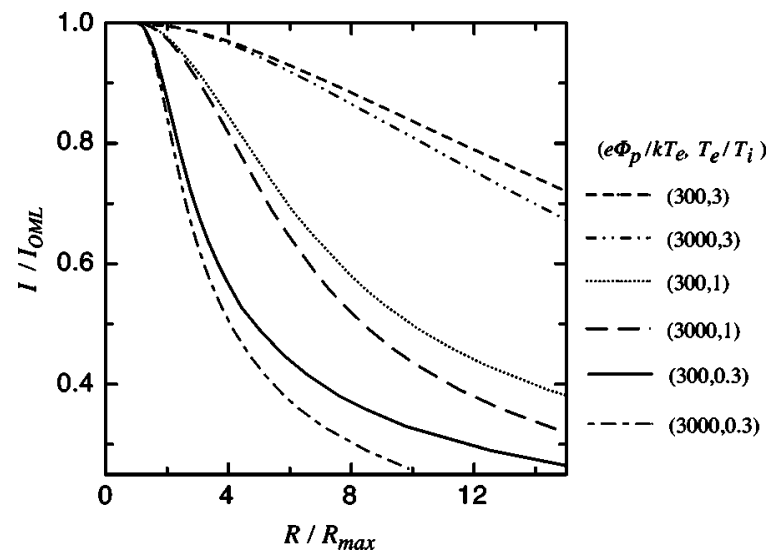

FIG. 5. Current ratio $I / I_{\mathrm{OML}}$ vs $R / R_{\max }$ for a few values of $e \Phi_{P} / k T_{e}$ and $T_{e} / T_{i}$.

proach is very slow; ${ }^{13}$ as a check, we found a value $I / I_{\mathrm{OML}}$ $=0.057$ at $R / \lambda_{\mathrm{De}}=393$ for $e \Phi_{P} / k T_{e}=1000$, and at $R / \lambda_{\mathrm{De}}$ $=209$ for $e \Phi_{P} / k T_{e}=3000$.

Note that curves in Figs. 4(a) and 4(b) can roughly be obtained from each other by a horizontal displacement that is $T_{e} / T_{i}$-dependent. Hence, the dependence on $R / \lambda_{\mathrm{De}}$ and $T_{e} / T_{i}$ can be approximated by a simple law that should be useful for design considerations,

$$
\frac{I}{I_{\mathrm{OML}}} \approx G\left(\frac{R-R_{\max }}{\lambda_{\mathrm{De}}}\right)=G\left(\frac{R}{\lambda_{\mathrm{De}}}-\widetilde{R}_{\max }\left(\frac{T_{e}}{T_{i}}\right)\right) .
$$

Here $\widetilde{R}_{\max } \equiv R_{\max } / \lambda_{\text {De }}$ is roughly a decreasing function of the ratio $T_{e} / T_{i}$, as determined in Ref. 9 , and $G$ is some universal function obtained from the figures $[G(0)=1, G$ decreasing with increasing positive argument]. Writing the argument of $G$ as $\left(R / R_{\max }-1\right) \times \widetilde{R}_{\max }$, it follows that $I / I_{\mathrm{OML}}$ drops faster with $R / R_{\max }$ the higher $\widetilde{R}_{\max }$, i.e., the lower $T_{e} / T_{i}$. This can be seen clearly in Fig. 5 , showing $I / I_{\mathrm{OML}}$ versus $R / R_{\max }$.

The present results lead to simple design conclusions: One might reasonably use tether radii over a sensible range beyond $R_{\max }$; this range exceeds $\lambda_{\mathrm{De}}$ even if $R_{\max }$ is well below $\lambda_{\text {De }}$ (the high- $T_{e} / T_{i}$ case); in terms of the ratio $R / R_{\max }$, the range increases rapidly with decreasing $R_{\max }$. These conclusions are relevant for standard applications of bare tethers, which find a plasma with Debye length and, to some degree, ratio $T_{e} / T_{i}$, varying along the orbit. These comments apply with even more force if the tether is used for orbit raising or lowering due to the large variations of plasma density with altitude.

The conclusions may also serve in discussing the effect of a plasma velocity $U$ relative to the probe. This introduces a new characteristic ion (ram) energy, which, for a tether orbiting in the $\mathrm{F}$ layer, where $\mathrm{O}^{+}$is the dominant ion species, is large compared with the thermal energy, $(1 / 2) m_{i} U^{2}$ $\approx 4.5 \mathrm{eV} \gg k T_{i} \sim 0.15 \mathrm{eV}$. Note that this is not the case at higher altitudes, where $\mathrm{H}^{+}$ions are dominant-and $U^{2}$ is somewhat reduced. In the F-layer the unperturbed ion distribution function would be strongly nonisotropic, and the electric field nonradial. 
The OML current law, however, is still valid, being independent of both ion distribution function and cross-section shape [just replace $2 R$ with perimeter $/ \pi$ in Eq. (1)]; ${ }^{8}$ the law does not require a rotationally symmetric potential. ${ }^{9}$ The law just requires that the unperturbed electron distribution function be isotropic, a condition well satisfied in our case, with $(1 / 2) m_{e} U^{2} \ll k T_{e}$; the high-bias limit law (1) is specially robust: It holds independently of that particular isotropic distribution. The effect of a large ion ram energy would then be a change in the domain of validity for the OML law.

We now recall that $I / I_{\mathrm{OML}}$ remains unity over some (and close to unity over a much larger) parametric domain, mirroring the fact that $I_{\mathrm{OML}} / I_{\mathrm{th}}$ in (1) is independent of $R / \lambda_{\mathrm{De}}$ and $T_{e} / T_{i}$. This means that one could alter substantially $T_{i}$, or the probe cross section (keeping its perimeter), thus fully modifying the structure of the potential field, without reaching the boundary of the domain of OML validity, that is, with no current variation. This is a case quite the opposite of large spherical electron collectors as used in conductive tethers previously flown. In predicting the new domain of validity (instead of an actual value for $I$ ) one might use crude models if conservative, although definite conclusions on this point must wait for a more careful analysis

For the conditions of interest, $e \Phi_{P} \gg(1 / 2) m_{i} U^{2}$, ions would be kept far away from the probe for all directions, with some (angle-dependent) potential structure similar to that shown in Fig. 1. In a crude model, one would ignore the nonthermal character of the ram energy, except for the fact that it makes the ion characteristic energy angle-dependent. In a plasma with $T_{i} \sim T_{e}$, one should have effective ion temperatures $k T_{i}(\mathrm{eff}) \sim(1 / 2) m_{i} U^{2} \sim 30 k T_{e}$ on the windward side, $T_{i}$ (eff) $\sim T_{e}$ on the lateral sides, and, as argued below, $T_{i}$ (eff) $\sim T_{e} \times \sqrt{2 k T_{e} / m_{i} U^{2}} \sim 0.2 T_{e}$, on the lee side. Figure 4(a), showing $I / I_{\mathrm{OML}}>0.95$ at $R=\lambda_{\mathrm{De}}$, for $T_{e} / T_{i}$ as high as 3 , then suggests that a probe of radius $R<\lambda_{\mathrm{De}}$ would collect current close to the OML value. Our estimate for $T_{i}$ (eff) on the lee side is based on the fact that, for other parameters fixed, the minimum distance reached by the ions, $r_{1}$ in Fig. 1 , depends on the characteristic ion energy; ${ }^{9}$ we then took $r_{1} \sim R \sqrt{e \Phi_{P} / k T_{i}} \sqrt{T_{e} / T_{i}}$ for $T_{e} / T_{i}$ large or about unity, from the zero- $U, R=R_{\max }$ analysis of Ref. 9, and $r_{1}$ (lee) $\sim r_{1}($ lateral $) \times \sqrt{m_{i} U^{2} / 2 k T_{e}}$ from simple wake considerations.

\section{ACKNOWLEDGMENT}

The work of J.R.S. was supported by the Comisión Interministerial de Ciencia y Technología of Spain under Grant No. PB97-0574-C04-1. The work of R.D.E. was supported by NASA Grant No. NAG8-1605.

${ }^{1}$ M. Martínez-Sanchez and D. H. Hastings, J. Astronaut. Sci. 35, 75 (1987).

${ }^{2}$ R. D. Estes, E. Lorenzini, J. R. Sanmartín, J. Pelaez, M. MartínezSanchez, L. Johnson, and I. Vas, J. Spacecr. Rockets 37, 205 (2000).

${ }^{3}$ R. D. Estes, J. Geophys. Res. 93, 945 (1988); J. R. Sanmartín and M. Martínez-Sanchez, ibid. 100, 1677 (1995).

${ }^{4}$ J. R. Sanmartín and R. D. Estes, J. Geophys. Res. 102, 14625 (1997).

${ }^{5}$ M. Martínez-Sanchez and J. R. Sanmartín, J. Geophys. Res. 102, 27257 (1997).

${ }^{6}$ J. R. Sanmartín, M. Martínez-Sanchez, and E. Ahedo, J. Propul. Power 9, 353 (1993).

${ }^{7}$ R. D. Estes, J. R. Sanmartín, and M. Martínez-Sanchez, J. Spacecr. Rockets 37, 197 (2000).

${ }^{8}$ J. G. Laframboise and L. W. Parker, Phys. Fluids 16, 629 (1973).

${ }^{9}$ J. R. Sanmartín and R. D. Estes, Phys. Plasmas 6, 395 (1999).

${ }^{10}$ L. W. Parker and E. C. Whipple, Jr., Ann. Phys. (N.Y.) 44, 126 (1967).

${ }^{11}$ Condition $E_{\text {env }}(r)=0$ in $(11 \mathrm{~b})$ corresponds to a minimum of $r^{2} \Phi$ the profile tangent at point 0 , when reached from above, crosses the origin in Fig. 1). The quasineutral solution has no such property reaching 0 from below, and breaks down there; use of the full Eq. (2), however, would locally round the profile with no effect beyond the immediate neighborhood of 0 , which just appears as the no energy-barrier point closest to 1 .

${ }^{12}$ Results for $R_{\max }$ from our analytical approach can be compared to numerical computations by Laframboise, who considered $e \Phi_{P} / k T_{e}$ of order unity or moderately large [National Technical Information Service Document No. 634596 (J. G. Laframboise, "Theory of spherical and cylindrical Langmuir probes in a collisionless, Maxwellian plasma at rest,' University of Toronto Institute for Aerospace Studies Report No. 100, 1966); copies may be ordered from the National Technical Information Service, Springfield, VA 22161]. Figures 42 and 50(b) in Laframboise's work give $R_{\max } \approx 0.96 \lambda_{\mathrm{De}}$ at $T_{e}=T_{i}$ and the highest bias, $e \Phi_{P} / k T_{e}=25$; Figs. 6 and 7 of Ref. 9 give $R_{\max } \approx 0.77 \lambda_{\text {De }}$, or about $20 \%$ too low, at the same conditions. Differences are greater at still lower bias, $e \Phi_{P} / k T_{e}=20$ or 15 .

${ }^{13}$ M. J. M. Parrot, L. R. O. Storey, L. W. Parker, and J. G. Laframboise, Phys. Fluids 25, 2388 (1982). 